\title{
GPPS-CH-2020-163
}

\section{NUMERICAL INVESTIGATION ON CORNER SEPARATION CONTROL IN A LINEAR COMPRESSOR CASCADE BY SYNTHETIC JET}

\author{
Yating Liu \\ Xi'an Jiaotong University \\ Email:yating1995@foxmail.com \\ Xi'an, Shaanxi China \\ Wenlin Huang \\ Xi'an Jiaotong University \\ Email:wenlin.h@yahoo.com \\ Xi'an, Shaanxi China
}

\author{
Zhiheng Wang \\ Xi'an Jiaotong University \\ Email:wangzhiheng@mail.xjtu.edu.cn \\ Xi'an, Shaanxi China
}

\begin{abstract}
Corner separation is a kind of three-dimensional separation which occurs commonly in the junction of the endwall and blade corner region of high-loaded compressor. The increases of flow losses and the blockage associated with the corner separation have negative effect on the efficiency and stability of compressor. In this paper the flow control of corner separation by a synthetic jet is numerically investigated with the Reynolds Averaged Navier-Stokes/Large Eddy Simulation (RANS/LES) hybrid method to improve the cascade performance under the design condition. The synthetic jet is mounted on the blade suction side of a subsonic linear compressor cascade using NACA65 blade profiles. The influence of the synthetic jet on the vortex structures and unsteady characteristics in the cascade is investigated. The results indicate that, with the presence of synthetic jet, the passage vortex is weakened, and the corner vortex is receded indirectly. The intensity and range of the concentrated shedding vortex of the suction surface boundary layer are reduced. The total pressure loss coefficient is decreased by $25.8 \%$ at the design operating condition. Through calculating the entropy generation rate in the cascade, it is found that the blade profile loss is responsible for the additional flow losses. The synthetic jet significantly reduces the irreversible losses in the blade passage and wake region. From the perspective of the bimodal distributions of corner separation, the parameters increase first and then decrease, and the bimodal points appear in the abrupt velocity zone rather than at the separation points only.
\end{abstract}

Keywords: Corner separation, synthetic jet, bimodal distributions, hybrid RANS/LES simulation

\section{INTRODUCTION}

Corner separation is one of the common complex flow phenomena in a high-loaded axial compressor, which affects the stability of compressor and restricts the further improvement of the aerodynamic performance of compressor ${ }^{[1]}$. In order to delay or weaken the corner separation and improve the aerodynamic efficiency and stability of compressor, researchers developed various flow control technologies for corner separation. The synthetic jet is an active flow control technology based on vortex motion, which synthesizes the turbulent shear flow by controlling the fusion of underdeveloped vortex coherent structures ${ }^{[2-4]}$. The synthetic jet promotes the transition of the laminar boundary layer or enhances the exchange of momentum between the fluid inside and outside the boundary layer, thereby achieving effective control of the flow separation. Compared with other forms of active control, the synthetic jet has the advantages of no complicated piping system, compact structure, low power consumption and light weight. Glezer et al. ${ }^{[2]}$ conducted the experiment on synthetic jet technology and found that the synthetic jet has great potential for controlling flow separation. Gmelin et al. ${ }^{[5]}$ used numerical and experimental methods to conduct the synthetic jet control on a high-loaded low-speed compressor cascade. The numerical simulation and experimental results are in a good agreement. Qin et al. ${ }^{[6]}$ took the low-speed and highspeed compressor cascades as the research objects, and numerically studied the effect of synthetic jets on the aerodynamic performance of the cascades. Based on the theory of vortex dynamics and topology analysis, the flow topology and vortex structure in the compressor cascades were analysed. 
It has been found that, the synthetic jet can delay the flow separation of the cascade and reduce the losses in cascade. However, the current understanding on the mechanism of synthetic jet controlling the corner separation is generally based on vortex structure analysis, and there is little excavation of the instantaneous velocity field. Ma et al. ${ }^{[7]}$ measured the corner separation of a low-speed compressor cascade and found that the probability density distributions of velocity on some measurement points are bimodal. These bimodal histograms can be decomposed into two Gaussian distributions. This is called by the bimodal histograms phenomenon, which indicates the strong intermittency and unsteadiness of the corner separation. Therefore, it is necessary to explore the changes of the bimodal phenomenon before and after the application of the synthetic jet for improving our understanding of the corner separation control mechanism by the synthetic jet.

In this paper, the SAS-SST turbulence model are used to simulate the corner separation in a linear cascade. The macroscopic characteristics such as total pressure loss and irreversible loss of corner separation flow will be studied before and after the synthetic jet control. In addition, the changes of vortex structure and the bimodal histogram are explored in this paper as well.

\section{NUMERICAL SIMULATION}

Research object and physical model

The research object of this paper is a low-speed linear compressor cascade. The detailed experiments have been performed by Laboratory of Fluid Transfer and Acoustics (LMFA) at Ecole Centrale Lyon such as in studies by Ma ${ }^{[7]}$ and Zambonini ${ }^{[18]}$. For the blade, its thickness distribution is the NACA 65-009 and its mean camber line is a circular arc with a camber angle of $23.22^{\circ}$.

Its main geometric and aerodynamic parameters are shown in Table 1.

\section{Table 1 Parameters of cascade parameters}

\begin{tabular}{llll}
\hline Chord & $c=150 \mathrm{~mm}$ & Aspect ratio & 2.47 \\
Camber angle & $23.22^{\circ}$ & Design upstream flow angle & $54.31^{\circ}$ \\
Stagger angle & $42.7^{\circ}$ & Incidence angle & $4^{\circ}$ \\
Axial chord & $c_{a}=110 \mathrm{~mm}$ & Inlet velocity & $U_{\infty}=40 \mathrm{~m} / \mathrm{s}$ \\
Pitch & $s_{p}=134 \mathrm{~mm}$ & Reynolds number & $R e=U_{\infty} c / v=3.82 \times 10^{5}$ \\
Span & $h=370 \mathrm{~mm}$ & & \\
\hline
\end{tabular}

In this paper, three components $s^{*}, n^{*}$ and $z^{*}$ are used to characterize the spatial position of a certain point in the cascade passage, as shown in Figure 1. A normalized parameter is used to indicate the actually travelled distance of the fluid along the suction side, defined as

$$
\begin{gathered}
s^{*}=s / L \\
n^{*}=n / s_{p} \\
z^{*}=z / h
\end{gathered}
$$

where $s$ is the length of arc from the leading edge to point A on the suction side, $L$ is the length of arc of the blade suction side, $n$ is the distance from a point in the cascade passage to the suction surface of the blade, and $z$ is the spanwise distance from a point in the cascade passage to the endwall.

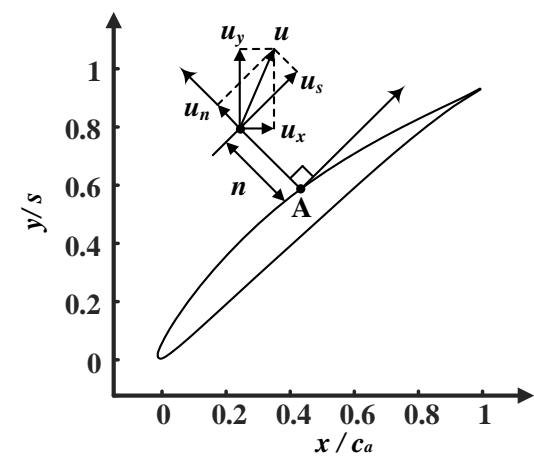

Figure 1 Velocity decomposition and $s, n$ coordinates

The synthetic jet is located on the suction surface of the blade, and the height of the slit in the span direction is $100 \% \mathrm{~h}$. The position of the jet outlet $s *$ is $70 \%$, the angle between the jet centerline and the local profile of the suction surface $\theta_{S J}$ is $30^{\circ}$, the inlet width of the actuator w is $0.5 \% c$, and the depth $h^{*}$ is $1 \% c$. 

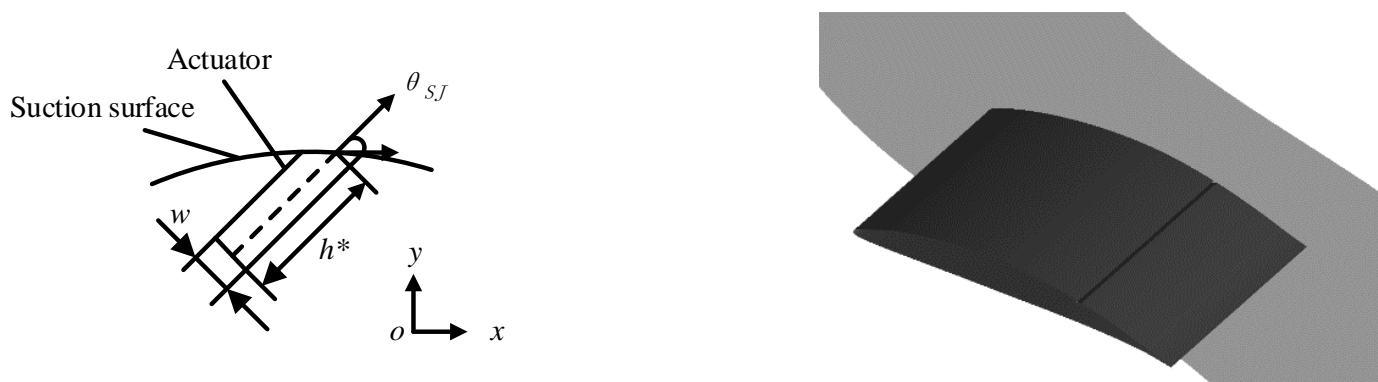

\section{Figure 2 Slit structure of synthetic jet actuator}

\section{SAS-SST turbulence model}

With the rapid development of computers, computational fluid dynamics (CFD) has become an important means of studying the corner separation. Among the turbulence models, the Reynolds-Averaged Navier-Stokes/Large Eddy Simulation(RANS/LES) hybrid method that takes into account the calculation accuracy and resource consumption is getting more attention ${ }^{[8]}$. The hybrid model uses the RANS model in the near wall area and the far field, and switches to a more reasonable LES-like filter model in the separation area, which greatly improves the calculation accuracy and reduces the calculation resource consumption.

Scale-Adaptive Simulation(SAS ${ }^{[8-9]}$ is a type of hybrid RANS / LES method. It uses the von Karman length scale $L_{\mathrm{vk}}$ that describes the local flow topology as the second length scale of the turbulence model. The construction method of the SAS-SST model is to add an adaptive source $\mathrm{Q}_{\text {SAS }}$ to the $\omega$ equation of the SST model, which is defined as

$$
Q_{S A S}=\max \left[\rho \xi \kappa S^{2}\left(\frac{L}{L_{v k}}\right)^{2}-C\left[\frac{2 \rho k}{\sigma_{\varphi}} \max \left(\frac{|\nabla \omega|^{2}}{\omega^{2}}, \frac{|\nabla k|^{2}}{k^{2}}, 0\right)\right]\right.
$$

where the turbulence scale $L=\sqrt{k} / C_{\mu}^{1 / 4}, L_{v k}=\kappa S /\left|\nabla^{2} U\right|$, model constant $\mathrm{C}=2.0, \quad \xi=3.51, \kappa=0.41, \sigma_{\varphi}=2 / 3, C_{\mu}=0.09$.

\section{Computational grid}

The numerical simulation was performed for the flow in a single blade passage with a half blade span. The computational domain is shown in Fig. 3 (a). The inlet of the domain is $2.5 \mathrm{c}$ away from the leading edge of the cascade and the outlet is $2 \mathrm{c}$ away from the trailing edge, which is consistent with the experiment ${ }^{[7]}$. In order to capture the flow field structure accurately near the boundary layer and the synthetic jet cavity, the grids near the wall and the slit are locally refined to ensure $y^{+}$is less than1.The total number of grid nodes before and after the synthetic jet was mounted are 5.4 million and 6 million, respectively.

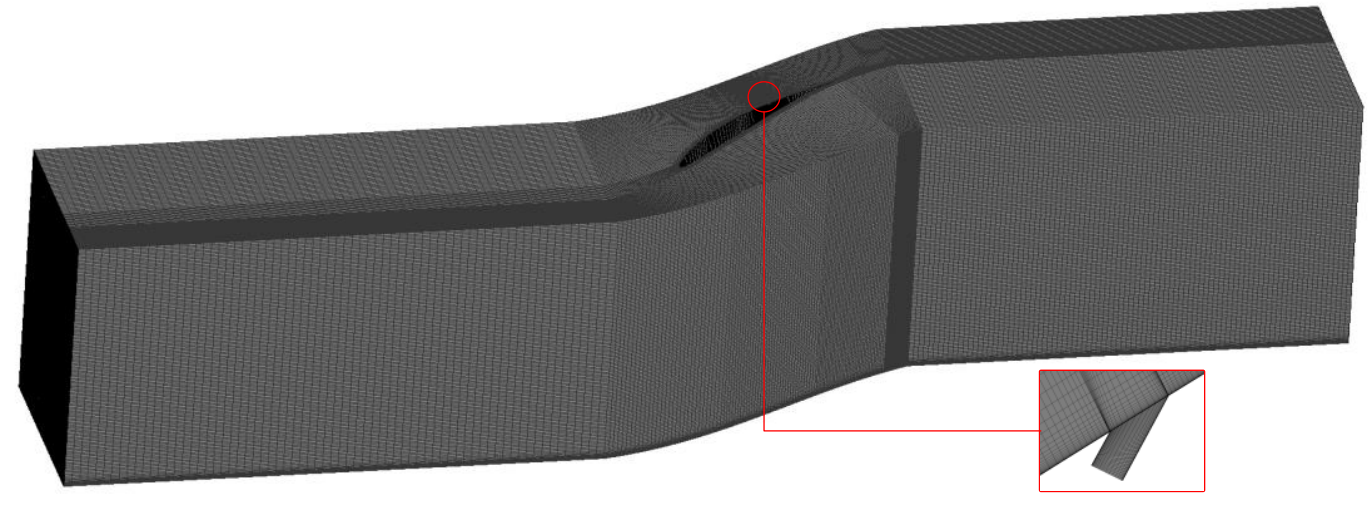

Figure 3 Computational domain and grid

\section{Numerical scheme and boundary conditions}

ANSYS CFX 14.0 was used for the numerical simulations in the paper. The SAS-SST model was chosen to simulate the computational domain. The viscosity term was calculated by the central difference format. The time term was solved iteratively using the second-order backward Euler scheme. The time step was 15 microseconds and the number of inner iterations for each time step was 10.

The inlet boundary condition was set as velocity boundary condition, and the inlet velocity profile was consistent with the experiment. The incidence angle is $4^{\circ}$. The turbulent dissipation rate and turbulent kinetic energy were prescribed based on the experimental data ${ }^{[7]}$. The outlet boundary condition was set to an atmospheric pressure. The blade surfaces and endwalls were assumed to be adiabatic. The middle span of the blade was set as symmetrical boundary condition. The boundary of the calculation domain in the circumferential direction was set as the periodic boundary condition. For the 
synthetic jet slot, the velocity boundary condition at slot inlet was given to simulate the periodic motion of the actuator diaphragm, which is defined as

$$
U_{S J}=U_{\max } \sin (2 \pi f t)
$$

where $U_{\max }$ is the maximum value of the jet velocity in a synthetic jet period, which is $40 \mathrm{~m} / \mathrm{s}$ in present study; $f$ is the excitation frequency, which equals to the characteristic frequency $f_{c}$ of the flow field in this paper, $f_{c}$ is defined as

$$
f_{c}=U_{\infty} / c
$$

\section{Verification of numerical simulation}

The experimental and numerical distribution of the static pressure coefficient on the blade surface is shown in the Figure 4. The static pressure coefficient is defined as

$$
C_{p}=\frac{p_{s}-p_{s \infty}}{p_{t \infty}-p_{s \infty}}
$$

where $p_{s}$ is the mean static pressure, $p_{s \infty}$ is the inlet mean static pressure, and $p_{t \infty}$ is the inlet mean total pressure. As shown in Figure 4 (a), for the main flow area (non-separation area), the SAS-SST numerical simulation results are consistent with the experimental results. However, as shown in Figure 4 (c) (d), for the separation area, the SAS-SST numerical simulation results deviate from the experimental results. Although there is a deviation from the experimental results, the SAS-SST is a compromise scheme: The more accurate method, LES, is more expensive ${ }^{[13]}$; The less costly URANS method cannot distinguish the vortex structures of corner separation well and deviate from the experimental results significantly ${ }^{[11]}$
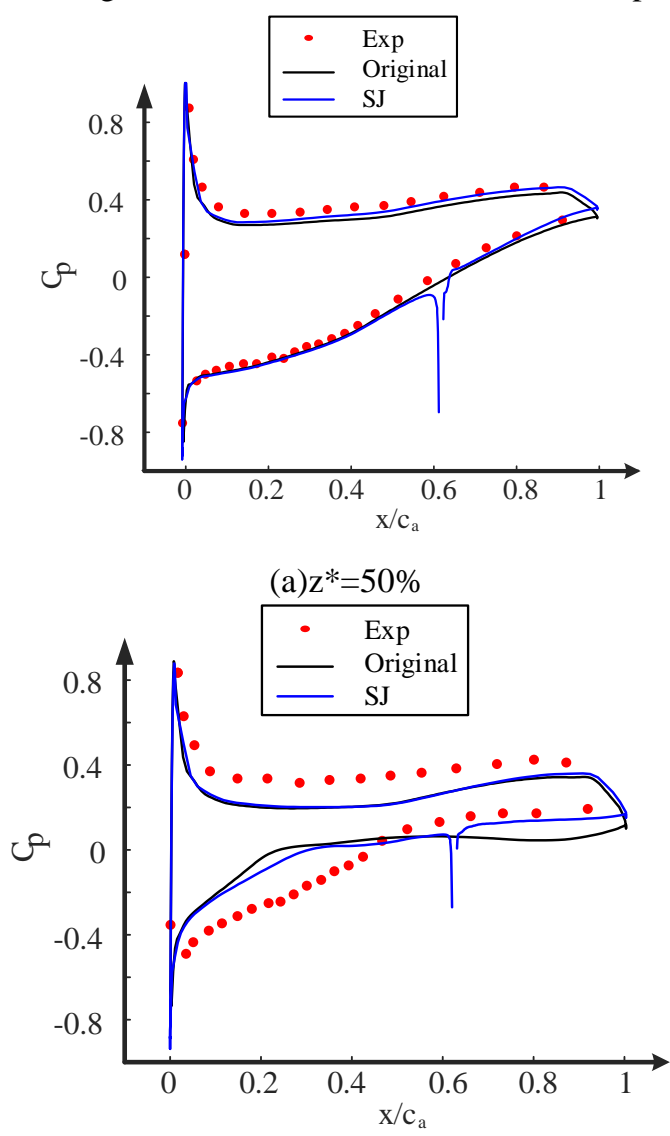

(c) $\mathrm{z}^{*}=5.4 \%$
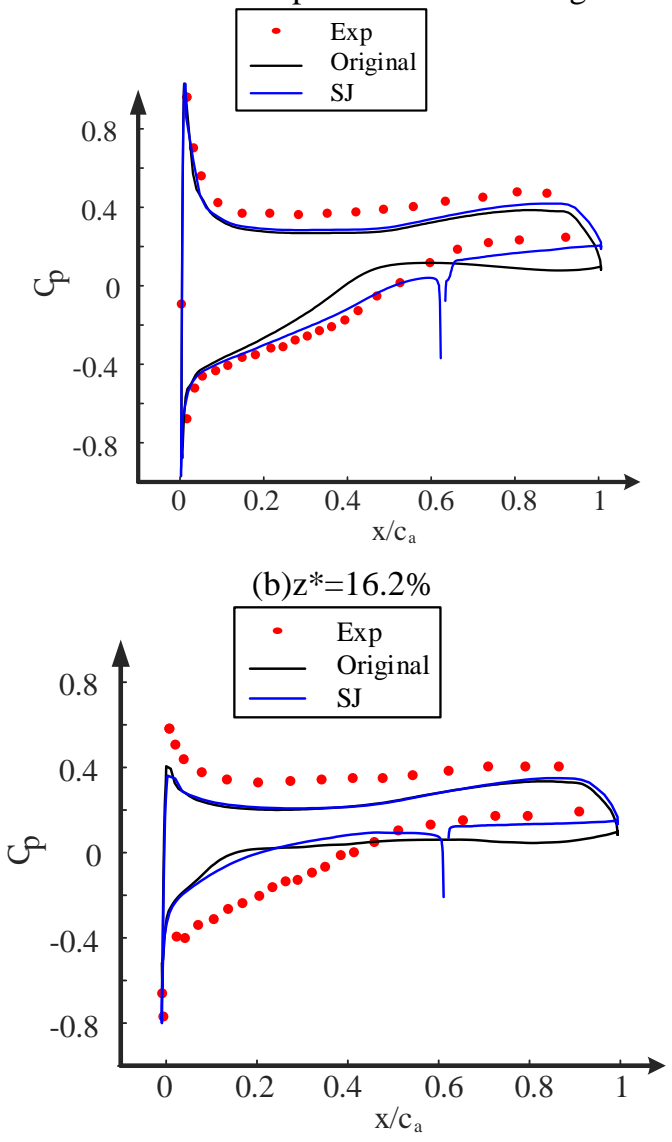

(d) $\mathrm{Z}^{*}=1.35 \%$

Figure 4 Static pressure coefficient of blade surface

In 2013, Ma et al ${ }^{[7]}$. measured the flow field of corner separation of the linear cascade channel. Through the Probability Density Function (PDF) analysis of the transient velocity measured by the Laser Doppler Anemometry (LDA), it is found that the velocity probability density distribution at many measuring points has two peaks, which are called bimodal points. Most of these bimodal points are located near the average separation line of the spanwise section. Ma successfully fitted the PDF of the transient velocity components of the bimodal points as the sum of two Gaussian distributions, indicating the existence of two random unsteady processes in the flow field. This phenomenon is known as the velocity PDF bimodal phenomenon in corner separation. Bimodal phenomenon reflects the strong intermittence of unsteady flow and indicates that there exists large-scale coherent structures corresponding to bimodal phenomenon in corner separation.

In the SAS-SST numerical simulation results, the gaussian decomposition of velocity probability density distribution of some points in cascade passage was carried out. The decomposition method is 


$$
P\left(u_{s}\right)=G_{1}\left(u_{s} ; a_{1}, U_{s 1}, \sigma_{u s 1}^{2}\right)+G_{2}\left(u_{s} ; a_{2}, U_{s 2}, \sigma_{u s 2}^{2}\right)
$$

where $G_{1}$ and $G_{2}$ respectively are

$$
\begin{aligned}
& G_{1}\left(u_{s} ; a_{1}, U_{s 1}, \sigma^{2}{ }_{u s 1}\right)=\frac{a_{1}}{\sqrt{2 \pi} \cdot \sigma_{u s 1}} \exp \left[-\frac{\left(u_{s}-U_{s 1}\right)^{2}}{2 \sigma_{u s 1}^{2}}\right] \\
& G_{2}\left(u_{s} ; a_{2}, U_{s 2}, \sigma_{u s 2}^{2}\right)=\frac{a_{2}}{\sqrt{2 \pi} \cdot \sigma_{u s 2}} \exp \left[-\frac{\left(u_{s}-U_{s 2}\right)^{2}}{2 \sigma_{u s 2}^{2}}\right]
\end{aligned}
$$

where, $a_{1}$ and $a_{2}$ represent the probabilities of $G_{1}$ and $G_{2}$, respectively, $U_{s 1}$ and $U_{s 2}$ are the mean of $G_{1}$ and $G_{2}$, respectively, $\sigma_{u s 1}^{2}$ and $\sigma_{u s 2}^{2}$ the variance of $G_{1}$ and $G_{2}$, respectively. The velocity Probability density distribution is decomposed into two Gaussian distributions with a similarity 0.983 to the original distribution, so it is reasonable to decompose into two Gaussian distributions. From the decomposition results, the SAS-SST numerical simulation can capture the bimodal histograms phenomenon, as shown in Figure 5. Under the same $s *$ and $z$ *values, as the value of $n *$ increases, $a_{l}$ and $U_{s 1}$ gradually increase, and $G_{1}$ and $G_{2}$ trade off each other. This trend is consistent with experimental results. Although the SAS-SST numerical simulation is different from the experimental results, it captures the unsteady characteristics of corner separation. Moreover, one of the purposes of this paper is to research the effect of synthetic jets on the unsteady characteristics of corner separation, the SAS-SST simulation results meet the analysis needs of this paper basically.

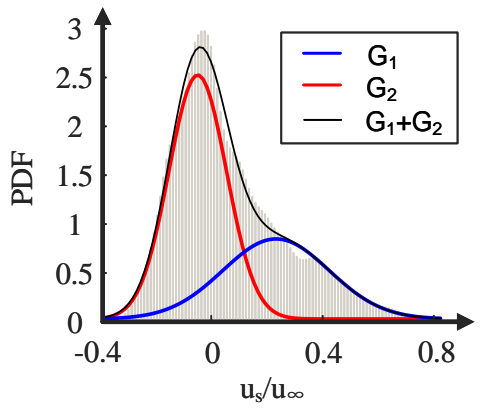

(a) $z^{*}=1.35 \%, s^{*}=50 \%, n^{*}=7.46 \%$

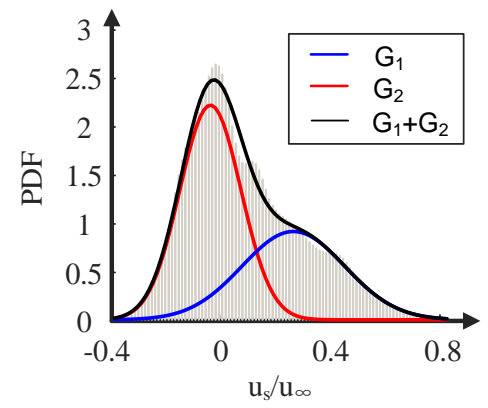

(b) $z^{*}=1.35 \%, s^{*}=50 \%, n^{*}=8.21 \%$

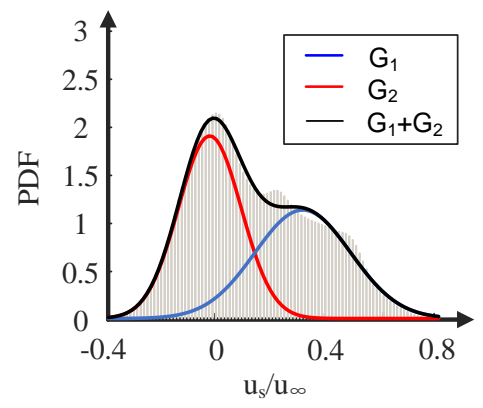

(c) $z^{*}=1.35 \%, s^{*}=50 \%, n^{*}=8.96 \%$

Figure 5 Bimodal histogram of SAS-SST simulation results

\section{Effect of synthetic jet on aerodynamic performance of cascade}

Effect of synthetic jet on time-averaged flow field

The distributions of averaged static pressure coefficient on the different spans before and after control are shown in Figure 4. With the presence of synthetic jet, there is a spike in the mean static pressure coefficient at the position of the actuator at different spans. The effect of the synthetic jet on the static pressure coefficient on the blade surface in the main flow region is negligible, which shows that the application of synthetic jet has no effect on the main flow. For the separation region, an obvious conclusion is that at different spans, the static pressure coefficient on the blade pressure surface is nearly unchanged, and the static pressure coefficient increases slightly near the trailing edge.

For the suction side, downstream of the synthetic jet slot, the static pressure coefficients of the blade at different spans are increased compared with that in the original case. Upstream of the synthetic jet slot, the trend of the static pressure coefficient on the suction surface is more stable than that in the original case, which indicates that after the application of synthetic jet, the separation range at these spans decreases. The pressure difference between the suction and pressure surface of the blade is reduced, which is helpful to reduce the secondary flow in the cascade passage.

The distribution contour of the time-averaged total pressure loss coefficient on the cascade outlet section is shown in Figure 6. The definition of instantaneous total pressure loss coefficient is

$$
w^{*}=\frac{m_{i n} p_{t \infty}+m_{j e t} p_{t j e t}-\left(m_{i n}+m_{j e t}\right) p_{t}}{m_{i n}\left(p_{t \infty}-p_{s \infty}\right)+m_{j e t}\left(p_{t j e t}-p_{s j e t}\right)}
$$

where $p_{\mathrm{s}}$ is static pressure, $p_{\mathrm{s} \infty}$ is the static pressure of the incoming flow, $p_{\mathrm{t} \infty}$ is the total pressure of the incoming flow, $m_{i n}$ is the inlet mass flow, $m_{j e t}$ is the mass flow of the actuator(for origin case, this item is 0 ), $p_{\text {sjet }}$ is the static pressure of the jet, $\mathrm{p}_{t j e t}$ is the total pressure of the jet. The calculation formula of the time-average total pressure loss coefficient is

$$
w=\frac{\int_{0}^{T} w^{*} d t}{T}
$$

Before and after control, there is a high-loss zone at the outlet section. After the synthetic jet is applied, the high-loss zone becomes narrower and longer, and the total pressure loss in the high-loss zone decreases. The mass-averaged total pressure loss coefficient on the section of $x / c_{\mathrm{a}}=163.64 \%$ is reduced by $25.8 \%$ with the synthetic jet. 


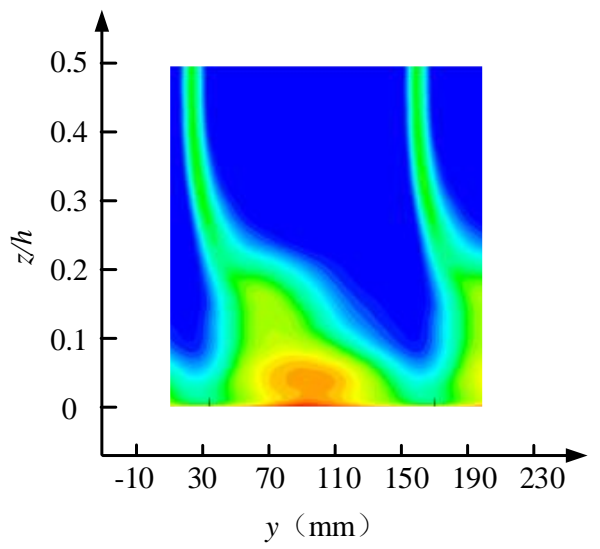

(a)Original case

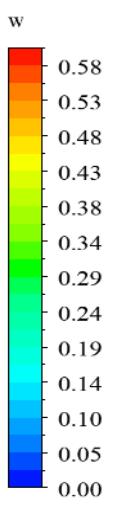

Figure 6 Total pressure loss coefficient at outlet section $\left(x / c_{a}=163.64 \%\right)$

The time-averaged limiting streamline on the blade suction surface is shown in Figure 7 . The synthetic jet does not change the flow characteristics and the position of the separation line on the suction surface upstream of the jet slot. For the original case, there are a concentrated shedding vortex and two separation lines on the blade suction surface. With the presence of synthetic jet, the concentrated shedding vortex disappears, and a saddle point appears near the endwall and downstream of the actuator. According to the critical point theory, there is a vortex structure at this saddle point. The intensity and scale of the vortex at this location are significantly smaller than the shedding vortex of original case. After the control, the starting point of the separation streamline near the endwall moves along the blade suction side toward the trailing edge. With the presence of synthetic jet, the downstream flow structure has been significantly improved, and the region of the separation has been significantly reduced. In addition, the size and strength of the vortex structure in the separation area was significantly reduced. The synthetic jet greatly improved the anti-separation ability of downstream of the blade suction surface.

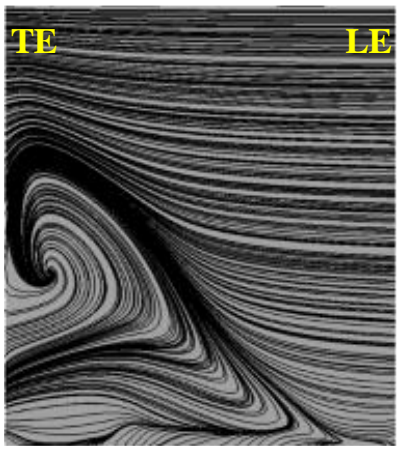

(a)Original case

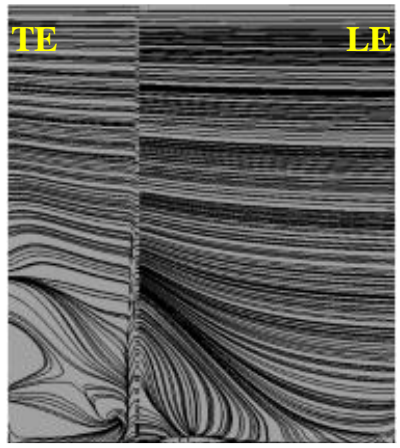

(b)SJ case

Figure 7 Time-averaged limiting streamline of suction side

To evaluate the local irreversible loss of flow field, the entropy generation rate due to viscous shear inside the viscous sublayer at the inlet was used to normalize the entropy generation rate over the entire flow fields ${ }^{[10]}$. The irreversible loss are divided into four parts: the wake-flow region loss, the endwall boundary layer loss, the blade profile loss and the bladepassage loss. With the synthetic jet, the loss of the whole simulation domain is reduced by $13.86 \%$. As shown in the Figure 8 , the blade profile loss is particularly significant. After the control, the loss of each part is reduced except the profile loss. The synthetic jet significantly reduces the irreversible losses in the passage and wake region, but the blade profiles loss increases. The synthetic jet removes the low momentum fluid on the suction side to avoid involving in the concentrated shedding vortex and corner vortex, but the addition of high-speed jets introduces additional irreversible losses. Due to the interaction between the corner vortex, the concentrated shedding vortex and the passage vortex are weakened, the aerodynamic performance of the blade passage and wake region is improved. 


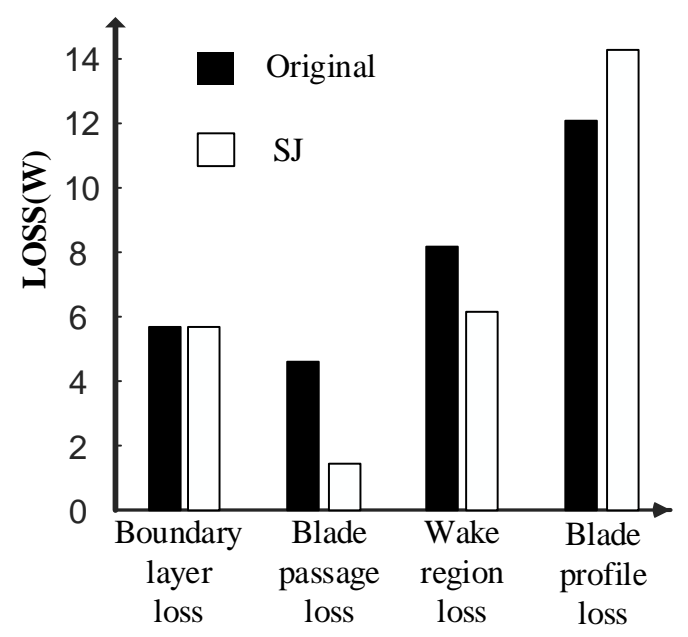

Figure 8 Irreversible loss analysis

The Q criterion is used to identify the vortex structure in the cascade passage, as shown in Figure 9. After the control, the scale of the shedding vortex and the passage vortex decreased significantly. The vortex at the outlet of passage also changed from large-scale vortex to small-scale vortex. After the control, the wake vortex formed a vortex street structure and it can also be seen that the intensity and influence range of the passage vortex and corner vortex are reduced.

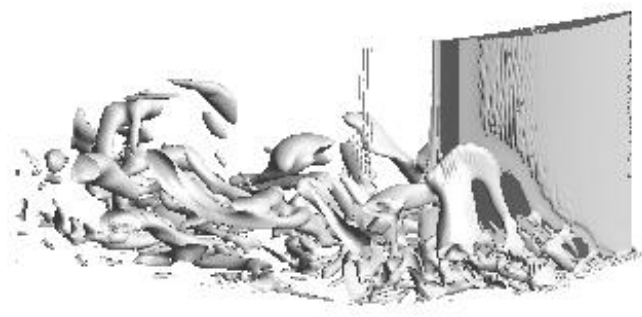

(a)Original case

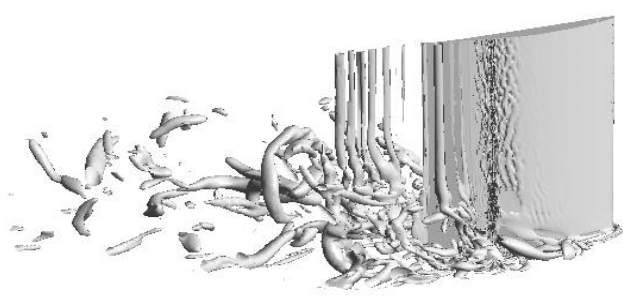

(b)SJ case

Figure 9 Vortex core region $\left(Q=10 U_{\infty} / c=7 \times 10^{6}\right)$

The time-average velocity vector diagrams at different blade heights sections are shown in Figure 10. Generally, when the time-averaged velocity is 0 , it is considered that the point is separated. Before the control, as the blade height $z^{*}$ increases, the separation point moves from the leading edge to the trailing edge, and the separation area gradually decreases. Before the control, the $z^{*}$ value of the edge of the separation area is greater than $16.22 \%$. After the control, the $z^{*}$ value of the edge of the separation area is between $8.11 \%$ and $10.81 \%$. At $z^{*}=1.35 \%, 2.70 \%, 5.41 \%$, although the separation point moves downstream after the control, at the same $s^{*}$, the separation area expands than origin case. Downstream of the actuator, a large range of backflow occurs. At $z^{*}=1.35 \%, 2.70 \%, 5.41 \%$, as the value of $n^{*}$ increases, the time-averaged velocity field upstream of the actuator increases first and then decreases after the control.

The positions of the bimodal points in the time-averaged velocity field are also shown in Figure 10. After control, the bimodal points generally appear in the velocity mutation region (velocity suddenly increases / decreases or changes from positive to negative). From the endwall to the middle-span section, the separation area gradually decreases, and the number of bimodal points also gradually decreases. Besides, the position of bimodal points gradually moves downstream towards the trailing edge. At the same blade height, the position of the bimodal points after control are generally closer to the leading edge than that in the original case. The velocity at the jet outlet fits to a sine function, so even in the non-separation area; there are bimodal points near the jet outlet. 

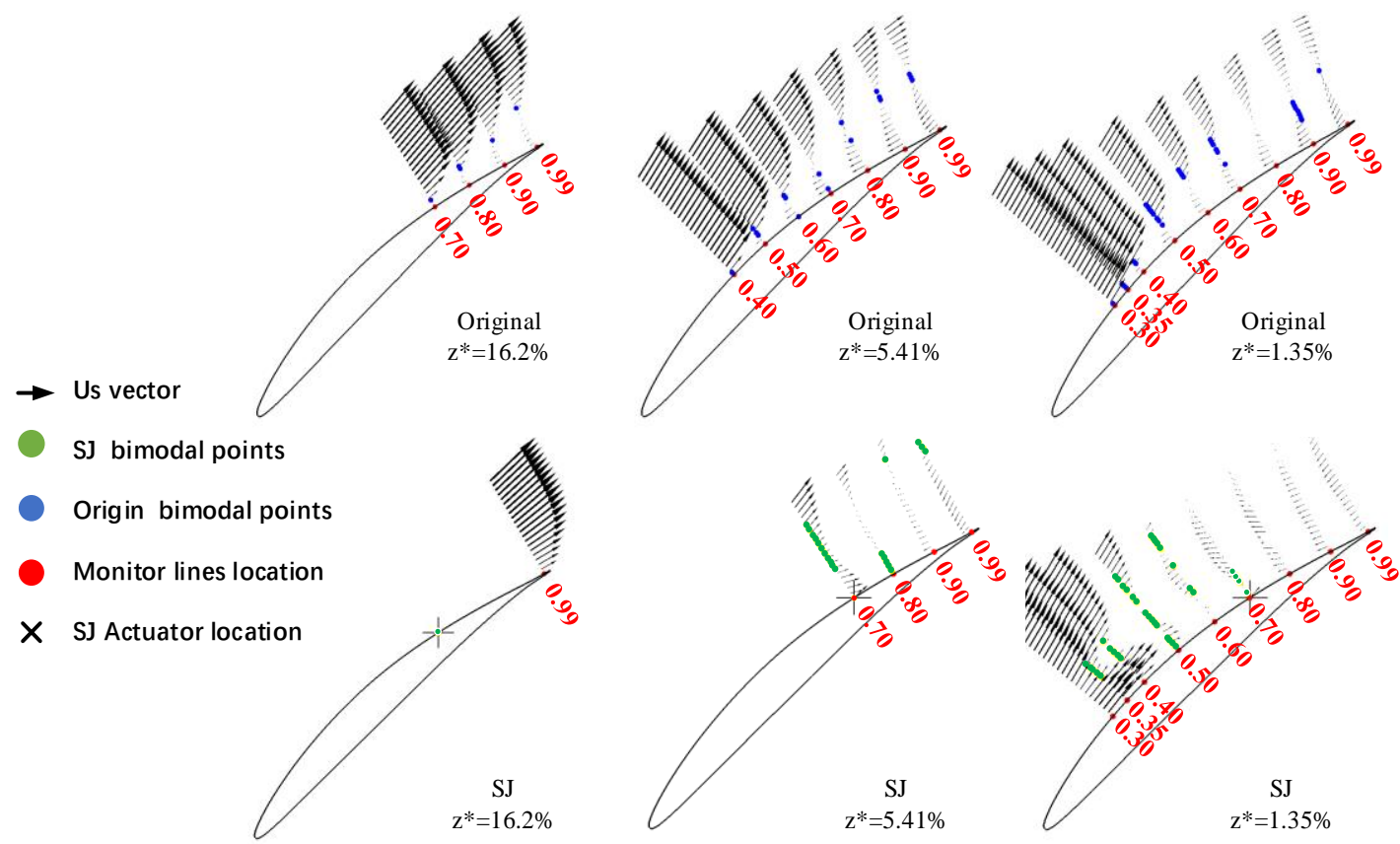

Figure 10 Bimodal points in mean velocity field

Instantaneous flow field characteristics before and after synthetic jet control

The frequency spectrums of the bimodal histogram velocity points after control are shown in Figure 11. Without control, there is no main frequency in the flow field. After control, the dominant frequency is found near the outlet of the jet slot. However, as the value of $n^{*}$ increases, the dominant frequency gradually disappears. There is no dominant frequency downstream of the slot, which indicates that the synthetic jet applied in this paper does not significantly affect the frequency of the flow field, and does not improve the intermittency of the flow field.

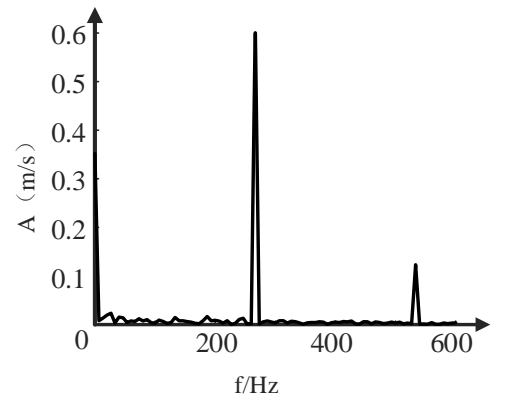

(a) $z^{*}=1.35 \%, s^{*}=70 \%, n *=0.75 \%$

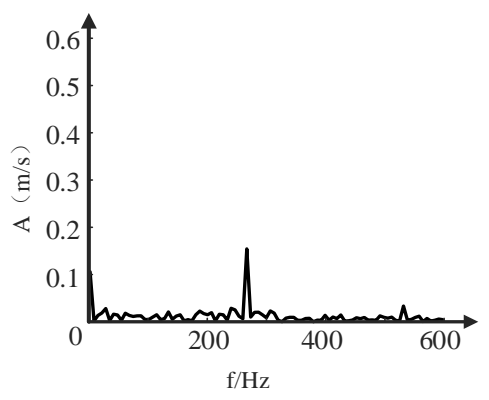

(b) $z^{*}=1.35 \mathrm{~mm}, s^{*}=70 \%, n *=1.49 \%$

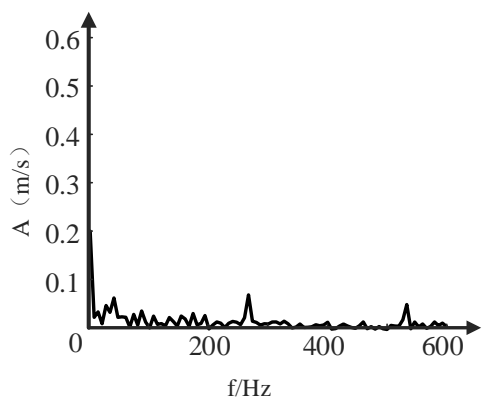

$(\mathrm{c}) * z=1.35 \%, s^{*}=70 \%, n *=7.46 \%$

Figure 11 Spectrums of velocity of bimodal points

Different from the original case, after control, when the value of $z^{*}$ is small $\left(z^{*}=1.35 \%, 2.70 \%, 5.41 \%\right)$, at the same value of $s^{*}$, as the value of $n *$ increases, $a_{l}$ increases first and then decreases, and $u_{s l}$ decreases first and then increases, ass shown in Figures 12 and 13.

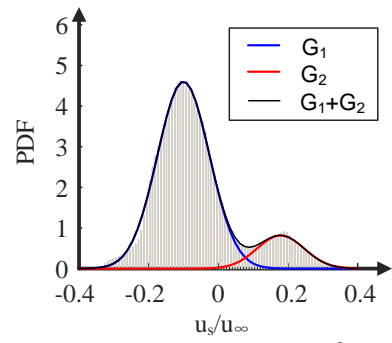

(a) $z *=1.35 \%, s *=60 \%, n *=9.70 \%$

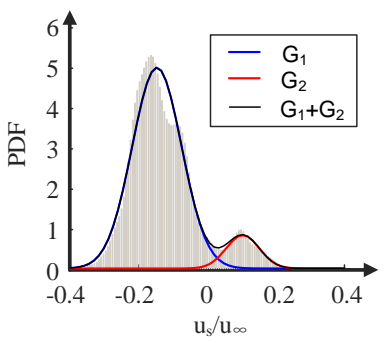

(b) $z *=1.35 \%, s^{*}=60 \%, n *=17.9 \%$

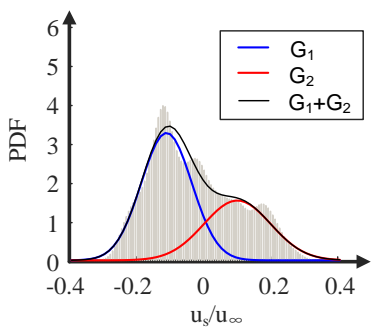

(c) $z^{*}=1.35 \%, s^{*}=60 \%, n^{*}=23.1 \%$

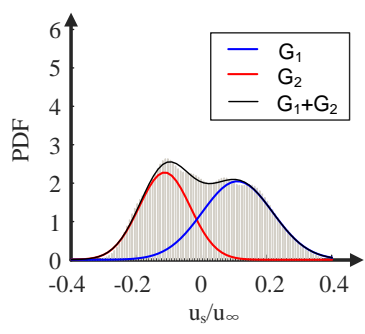

(d) $z^{*}=1.35 \%, s^{*}=60 \%, n^{*}=24.6 \%$

Figure 12 Bimodal histograms of SJ case 


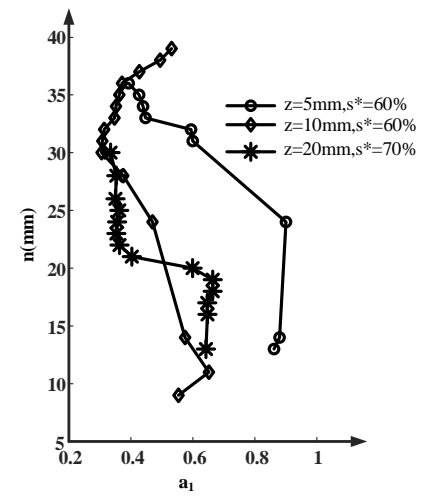

(a)Distributions of $a_{1}$ at bimodal points

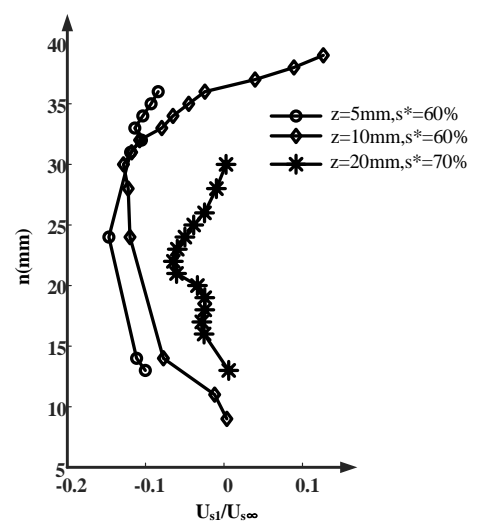

(b) Distributions of $U s_{1}$ at bimodal points

Figure 13 Bimodal parameters at different points

\section{Conclusions}

In this paper, the flow control of corner separation by a synthetic jet on blade suction side is numerically investigated with hybrid LES/RANS method to improve the linear cascade performance under the design condition.

The synthetic jet on the suction side improves the aerodynamic performance. The separation zone is significantly reduced. The total pressure loss coefficient is decreased by $25.8 \%$ at the design operating condition. The irreversible loss is mainly caused by the blade profile loss. The synthetic jet removes the low momentum fluid on the suction side to avoid involving in the concentrated shedding vortex and corner vortex, but the addition of high-speed jets introduces additional losses. Therefore, the intensity and range of the shedding vortex decreases, and the irreversible loss is reduced. In addition, the intensity and influence range of the passage vortex is also reduced.

The addition of the synthetic jet changes the unsteadiness and intermittent of the separation. With the synthetic jet, the bimodal points appear in the abrupt velocity zone rather than at the separation points only. Additionally, the parameters $\left(U_{\mathrm{s} 1}, a_{1}\right)$ increase first and then decrease instead of monotonically increasing. However, the presence of the synthetic jet in this paper does not significantly affect the frequency of the flow field.

\section{Acknowledgement}

The work is financially supported by National Nature Science Foundation of China (Grant No. 51576153) and the Key Research and Development Program of Shaanxi in China (Grant No. 2018KW-017), which are highly acknowledged.

\section{References}

[1] Gbadebo S A. Three-Dimensional Separations in Compressors[D]. Cambridge, UK: University of Cambridge, 2003.

[2] Glezer A, Amitay M. Synthetic Jets[J]. Annual Review of Fluid Mechanics, 2002, 34(1): 503-529.

[3] Smith D R, Amitay M, Valdis K, et al. Modification of Lifting Body Aerodynamics Using Synthetic Jet Actuators[C]. 36th AIAA Aerospaces Science Meetting and Exhibit, Reno, Nevada, 1998, AIAA 98-0209.

[4] Amitay M, Glezer A. Aerodynamic Flow Control of a Thick Airfoil Using the Synthetic Jet Actuators[C]. Prcoceedings of 3rd ASME/JSME Joint Fluids Engineering Conference, San Francisco, California, 1999.

[5] Gmelin C, Zander V, Hecklau M, et al. Active Flow Control Concepts on a Highly Loaded Subsonic Compressor Cascade: Résumé of Experimental and Numerical Results [J]. ASME Journal of Turbomachinery, 2012, 134(6): 061021.

[6] Qin Y, Song Y, Chen F, Wang R, Liu H. Active Flow Control by Means of Endwall Synthetic Jets on a High-Speed Compressor Stator Cascade[J]. Proceedings of the Institution of Mechanical Engineers, Part A: Journal of Power and Energy, 2017.

[7] Ma W,Ottavy X,Lu L,et al. Intermittent Corner Separation in a Linear Compressor Cascade[J]. Experiments in Fluids, 2013, 54(6): 1-17.

[8] Menter F R, Kuntz M, Bender R.A Scale-Adaptive Simulation Model for Turbulent Flow Predictions [R].AIAA2003-767.Reston:AIAA, 2003.

[9] Menter F R, Egorov Y. A Scale-Adaptive Simulation Model Using Two-Equation Models [R].AIAA-20051095.Reston:AIAA,2005.

[10] Li Z, Du J, Ottavy X, Zhang H. Quantification and Analysis of the Entropy generation Flow Loss in a Linear Compressor Cascade[J]. Entropy, 2018, 20(7): 486.

[11] Gao F, Ma W, Jérôme B, et al. Numerical Analysis of Three-Dimensional Corner Separation in a Linear Compressor Cascade[C]. Proceedings of ASME Turbo Expo 2013: Turbine Technical Conference and Exposition, San Antonio, Texas, 2013. 
[12] Ma W , Gao F, Ottavy X, et al. Numerical Investigation of Intermittent Corner Separation in a Linear Compressor Cascade[C]. ASME Turbo Expo: Turbomachinery Technical Conference \& Exposition. 2016:V02AT37A035.

[13] Gao F, Ma W, Sun J, et al. Parameter study on numerical simulation of corner separation in LMFA-NACA65 linear compressor cascade[J].Chinese Journal of Aeronautics,2017,30(01):15-30.

[14] Matejka M, Popelka L, Safarik P, et al. Influence of Active Methods of Flow Control on Compressor Blade Cascade Flow[C]. Proceedings of ASME Turbo Expo 2008, Berlin, Germany, 2008, GT2008-51109.

[15] Gmelin C, Steger M, Thiele F, et al. Unsteady RANS Simulations of a Highly Loaded Low Aspect Ratio Compressor Stator Cascade with Active Flow Control[C]. Proceedings of ASME Turbo Expo 2010, Glasgow, UK, 2010, GT2010-22516.

[16] Gmelin C, Steger M, Zander V, et al. Numerical Investigations of Active Flow Control Using Synthetic Jets on a Highly Loaded Compressor Stator Cascade[C]. Proceedings of the ASME 2010 FEDSM-ICNMM, Montreal, Canada, 2010, 2010-30725.

[17] Zander V, Hecklau M, Nitsche W, et al. Active Flow Control by Means of Synthetic Jets on a Highly Loaded Compressor Cascade[J]. Proceedings of the Institution of Mechanical Engineers, Part A: Journal of Power and Energy, 2011, 225(7): 897-906.

[18] Zambonini G, Ottavy X. Unsteady Pressure Investigations of Corner Separated Flow in a Linear Compressor Cascade[C]. Proceedings of ASME Turbo Expo 2016: Turbomachinery Technical Conference and Exposition, Seoul, South Korea, 2016. 